\title{
A HOLISTIC FRAMEWORK FOR INFORMATION SYSTEM FOR THE MANAGEMENT OF INDOOR ENVIRONMENTAL QUALITIES (IEQ) IN BUILT FACILITIES
}

\author{
Ahmed M. Ibrahim ${ }^{1}$, Mohammad A. Hassanain ${ }^{2}$ and Ibrahim A. Hameed ${ }^{1}$ \\ ${ }^{1}$ Department of ICT, Norwegian University of Science and Technology, Ålesund, Norway \\ ${ }^{2}$ Architectural Engineering Department, King Fahd University of Petroleum and Minerals, Dhahran, Saudi Arabia
}

\begin{abstract}
Because every building is considered as a long-run investment, regardless of its scale, at least to its owner, broadly by collectively impacting the national development of built assets. It is a vital aspect to ensure the qualities which define its inhabitance experience. Buildings' indoor environments have been undermined being observed as an uncontrolled expensive application of systems, targeted for research, while indoor environmental quality (IEQ) is one of the assembly of aspects that aim to appraise almost any built facility performance. There have been research efforts in literature, with global and contextual limitations, covering dispersed relations and topics in the IEQ domain, such as the control, and analysis of occupants' satisfaction and facilities systems performance in respect to standards. These research efforts were more focused on investigating results rather than allowing for an automated solution for the creation of universal standards. Therefore, emerged a need for modelling IEQ management in an inclusive manner, to allow for the sustainable management and understanding of facilities components' impacts, on both occupants' experiences and wellbeing, whereas the power of information systems with the capabilities of todays' hardware and technologies, set a new era, for the architectural science in digital means. This research paper proposes a conceptual information system framework modelled in Integrated Computer Aided Manufacturing Definition for Function Modeling $\left(\mathrm{IDEF}_{0}\right)$ diagram, to enable the architects and the facility managers to sustainably manage and monitor IEQs in built facilities. The proposed framework is based on a standardized big data analytics that can provide architects and facility managers with meaningful insights for achieving an evidence based, and informed design thinking, as well as sustainable monitoring of built facilities in real time.
\end{abstract}

\section{KEYWORDS}

Framework, IEQ, Information System, Open Database, Big Data Analytics, Universal, Facilities Management, Life Cycle Assessment (LCA), Sustainable Development Goals (SDGs)

\section{INTRODUCTION}

Facilities management is the science which encompasses people, process and technology endeavoring highest quality of occupation, services, and operation of the built environment. Built facilities are considered as a long run investment by their owners, (either as organizations or individuals), that need to maintain satisfactory conditions for its indoor environments. The challenge is in alignment of users' satisfaction, while maintaining control over the variety of systems in facilities, through the continuous appraisal of operational performance, on the macro-urban and micro-indoor environments (Alexander, 1992; Bröchner et al., 2019; Lindkvist et al., 2020).

The advancement of facilities is evaluated by benchmarking of facilities with similar functionalities, in different indoor environmental quality (IEQ) elements that are not limited to the air cooling, heating, ventilation, conditioning, thermal comfort, indoor air quality (IAQ), spaces acoustics, illumination, aesthetics and recently to the exposure of electromagnetic frequencies (Dan Norbäck, 2020; Ribino et al., 2020; Wei et al., 2020). Unsatisfactory conditions impact occupants by several means, such as sick building syndrome, noise, contamination, thermal discomfort, and loss of productivity (Hameen et al., 2020). Thus, many researchers worked on different research objectives, by focusing on relational impacts of building systems 
design, selection and operation on occupants. In a sense where IEQ is put into question, from a holistic point of view.

International organizations, e.g. American Society of Heating, Refrigerating and Air-Conditioning Engineers (ASHRAE) provides an international standard based on collected global data, to develop a thermal comfort database. Unfortunately, the limitation was always geographical, varying based on the providing countries systematic research and collection methods, undertaking IEQ data. The resulting database was in excel format and limited to specific regions (such as: Australia, Belgium, Brazil, Canada, China, Denmark, France, Germany, Greece, India, Indonesia, Italy, Japan, Malaysia, Mexico, Nigeria, Pakistan, Philippines, Portugal, Singapore, Slovakia, South Korea, Sweden, Thailand, Tunisia, UK and USA). Furtherly the database included a limited number of indicators and facilities, Yet, considered to be one of the most comprehensive data for designers and facility managers (Földváry et al., 2018). Other organizations such as Chartered Institution of Building Services Engineers (CIBSE), U.S. Green Building Council (USGBC), and online benchmarking tools also developed an emerged sources of data but with geographical and contextual limitations, serving only specific focus on subjective or objective measures, that lack holisticness in the making (Heinzerling et al., 2013).

What if there is an enabling platform, along with the advancement of technology, that can be implemented at a wider prospect, openly over the planet, without commercial interests, that can release precise and interoperable standards for all. Allowing designers to an access for a globalized standard, where engineers from a specific region can open access standards for another, by then, newer business opportunities would emerge in an accelerating world of demands.

There are different standards that developed indices and requirements to maintain satisfactory indoor environment, based on research conducted at different facilities, such as: Center for the Built Environment at Berkley, Center for Sustainable Built Environment at New York University, and Centre for a Sustainable Built Environment at KTH royal institute of technology. Despite the innovative tools developed by such organizations, usually, the process of developing such standards and recommendations holds time, comprise extensive costs for funding, to measure, review and analyze built environment IEQ data, as well as they do not deliver an open and universal database, that allows for an accustomed analyses, of a longtime historical and accurate ranges, that can effectively enhance the understanding, design and operation of the indoor environments of facilities. Different tools and formulae can be used for measuring IEQ indices, these organizations have made a distinguished advancement being able to collect specific features about the space. The collected measurements and calculation of IEQ indices allow architects and facility managers to analyze evidence for future design projects and operation of existing facilities. Different regions have developed own standards, to promote guidelines for designers and facility managers to improve its indoor environment targeting sustainable design and operation of facilities. With time the standards developed serve to enrich a knowledge base that contains analyzed data in various formats.

Since, people spend $90 \%$ of their time in built facilities (Danza et al., 2020) being critical to their wellbeing. Thus, the dispersed research efforts in literature establish research questions, that needs to be investigated.

\section{RESEARCH AIMS AND QUESTIONS}

- How can IEQ indicators be updated systematically in an agile and collective manner?

- How can the IEQ be assessed in a holistic manner? combining physical measurements and subjective feedbacks from the users about their built environment?

- How can IEQ be assessed to serve a universal geographical context allowing for watch over different types of facilities?

- How can IEQ serve the sustainability and operation of facilities?

- How can IEQ data be processed to provide a consistent, and an on-demand reporting?

- What are the potentials of a holistic IEQ database? for researchers, designers as well as regulators? 


\section{RESEARCH METHODOLOGY}

The research is designed to comprise of three main stages:

1) Review of select of recent journal publications in the literature, within the domain of facilities management and IEQ research, on the holisticness of approaches for the assessment and management of built facilities, questioning for the evaluation tools, techniques, and methods producing the IEQ indicators. Also, to review the literature upon geographical contexts and buildings typologies coverage for IEQ research.

2) Development and proposal of a systematic framework allowing for a universal IEQ data collection, assessment, reporting, benchmarking. This is for allowing the sustainable operation and maintenance of built facilities and keeping of design recommendations of new facilities.

3) Conclusion and recommendations for the proposed framework and further considerations that can lead to effective validation.

\section{LITERATURE REVIEW}

Scopus search engine was utilized to synthesize journal publications in 2020, on the topic of IEQ. Keywords searched for were: "Indoor environmental quality framework", "IEQ", "Assessment of IEQ" and "IEQ benchmarks". A plethora of research results emerged in the database, yet, most of the references focused on investigating relational performance elements between demographics of the occupants and the IEQ, while some references focused on the development of compass tools for visually representing the IEQ indicators, other references focused on utilizing different measurement devices for physical and subjective measurements of IEQ performance, Yet the references did not serve the need for a holistic framework for the management of IEQ, on a high level of processes. Fast readings of the papers' contexts were conducted to synthesize recent references that are critical to provide an overall impression of the research magnitudes, contexts as in the literature on IEQ, the review findings were as follows:

Ioannou et al. (2019) researched the possibility of using a free software to calculate a prediction for heat strain of individuals at a built facility (based on equations and formulae elaborated in ISO 7933:2018). This is for optimization of calculating the heat strain, the developed software model is namely: FAME Lab PHS Calculator software $\left(\mathrm{PHS}_{\mathrm{FL}}\right)$. The researchers reviewed further related standards of different regionality. The validation of the software has shown high predictability in accordance with standards by $95 \%$ significance. This finding promotes the potential behind the historical IEQ data and its collection accuracy to predict a facility IEQ indicator, and report it in accordance to different standards. The authors discussed that "it is not always possible to perform the necessary physiological assessments in workers due to, for instance, lack of equipment, expertise, resources, time, and/or inability to recruit volunteers". Despite this statement, A project by the European Union was funded to develop a model for predicting heat strain (PHS) over a group with known environmental and physiological conditions. Based on dynamically variant inputs and parameters, the researchers discovered high significance, by comparing predicted results to field experiments. Such predictability, enables physiologists, industrial hygienists, and occupational physicians to enhance workers' health and improve productivity by improving the built environment.

Hassanain (2007) evaluated IEQ by conducting an indicative Post Occupancy Evaluation (POE) on students housing facilities, at a public university in Saudi Arabia. The research involved investigations of historical maintenance data, frequent complains and areas of discomfort raised by the occupants, as well as the conduct of surveys to obtain their feedback qualitatively. The analyses lead to recommendations directed for designers, planners and operation and maintenance teams upon satisfactory requirements for similar type of facilities projects (future students' housings). The elements investigated were an IEQ requirements of thermal, acoustical, visual comfort, and IAQ. Several standards were referenced to benchmark the facilities existent conditions. The research involved processes of data analyses, were plans of the facility were referenced to provide a schematic understanding of design impacts in relevance to occupation. An overall satisfaction mean was calculated, to represent the sampled opinions. The research was produced in a manner, that it can be applied to a similar type of educational housing facilities, especially in Saudi Arabia. 
Cao et al. (2020) researched the impacts of Heating Ventilation and Air Conditioning (HVAC) system and controls, on wellbeing of occupants. The research highlighted the role of IEQ investigations, in relevance to Chinese standards of performance criteria. This was initiated by a promoting requirement in china to induce passive elements in facilities, that can allow for the realization of near-zero energy buildings. The authors highlighted that HVAC systems can be responsible for more than 50\% of a total energy consumption in built facilities. Moreover, because that HVAC can directly affect the IAQ, where pollutants transferability is a subject of concern. The IEQ Elements under investigations were Particulate matters with different sizes such as $\left(\mathrm{PM}_{10}\right.$, and $\left.\mathrm{PM}_{2.5}\right)$, Volatile Organic Compounds $\left(\mathrm{VOC}_{\mathrm{s}}\right)$ and Nitrogen oxides $\left(\mathrm{NO}_{\mathrm{x}}\right)$, Airborne fungi, Ozone $\left(\mathrm{O}_{3}\right)$, radon and Carbon dioxide $\mathrm{CO}_{2}$ concentrations. Since pollutants have undesirable impacts on human health by impacting the overall wellbeing of occupants, thus, its needed to understand the correlations, allowing better regulation of air to enhance quality and reduce such risks. Thus, the study researched correlation between different IEQ and HVAC systems performance under the check of compliance to Chinese specifications, for the better understanding of the systems performance. The research recommended the need for establishing a systematic feedback mechanism by regulation, to assess the correlation between the environmental control and human behaviors.

Yoseph (2020) investigated a set of parameters in residential built facilities, located at Borg el Arab region in Egypt. This is to relate the IEQ design elements and performance with urban formation of units, for future developments of residential urban communities and evaluation of the regulatory standards comprehensiveness. The author discussed several buildings characteristics that affected the IEQ, along with the design of openings in each unit (e.g. orientation, size, and positioning). The spatial and environmental standard of Egypt was assessed, to validate performance of the facilities. A parametric simulation performed identified links between microclimate parameters (exterior of the building) and IEQ (interior of the building). The study highlighted that, despite satisfactory design of the units to the legislations, there is still a need to update the impacts of the urban morphology on the IEQ.

Geng et al. (2020) investigated intensity of energy use (EUI) in link to the performance of IEQ, for elements such as: thermal comfort, air quality and visual environment. The research encompassed a synthesis of three million sets of environmental data, and a relevant sample of 1,756 users' satisfaction surveys. The findings indicated that users were less satisfied about the thermal comfort and indoor air quality in facilities with high EUI due to the limitations of environmental control, in compare to users within low EUI facilities. Furtherly, facilities with high EUI scored higher conformity to local standards more than facilities with low EUI, while both groups of facilities seemed to confirm similarly to local standards in aspects of visual performance. The findings of this study indicated that, the investigated, green facilities suffered from overcooling and overheating, due to the minimal conformity to thermal comfort requirements. Furtherly, the study introduced an environmental energy efficiency indicator, for a linked evaluation of IEQ performance and energy consumption comprehensively, claiming that IEQ of a facility should not contradict with the energy efficiency. Thus, the authors reviewed IEQ and Energy use in literature and reported negative energy performance of green buildings and concluded that LEED buildings did not save energy compared to non-LEED buildings. Furtherly, they highlighted that there was a gap in literature focusing on this correlation in different regional localities.

Danza et al. (2020) discussed the challenge of zero energy buildings (ZEN), to deliver a quality of IEQ. The authors referred that there is not a unified standard for the comprehensive evaluation of IEQ. The reviewed literature indicated that research cases are dispersed in their findings and there is a need to rank importance of several factors affecting IEQ, in the built environment. Thus, the authors carried investigative surveys on 100 occupants of different ZEN buildings, to solicit their levels of comfort and satisfaction. Furtherly, physical measurements were taken in specific ZEN facilities conditions. The analyses of the surveys led to ranking of several indoor environmental factors. The authors highlighted the need to investigate the same indicators over different ZEN facilities with varying conditions. As research was conducted over a short exposure time, it was considered a limitation for the findings. A longer exposure time of sensors collecting measurements information and users' satisfaction data, would alter the research results and findings, to be more stable against wider fluctuation in environmental conditions.

Kishi and Araki (2020) discussed the need for improvement of IEQ as they are directly linked to sustainability. As built facilities design, where characteristics of a building such as increased air tightness can lead to symptoms of a sick home syndrome (SHS); according to a case from japan. The symptoms of SHS are not limited to sensory irritation, headache, dry cough, nausea, dermal itches, and dizziness. 
Since, health of occupants in a built facility is linked to the controls over its IEQ performance, a healthy indoor environment can achieve the realization of many of United nations (UN) sustainability development goals (SDG), such as:

- SDG 3 of maintaining good health and wellbeing.

- SDG 5 of gender equality.

- SDG6 of clean water and sanitation.

- SDG7 Affordable and clean energy.

- SDG 8 decent work and economic growth.

- SDG 9 industry, innovation, and infrastructure.

- SDG 10 sustainable cities and communities.

- SDG 12 of responsible production and consumption.

- SDG 13 climate action.

IEQ is related to wellbeing of occupants, e.g. understanding the functional use of spaces and variation of men and women, such as exposure to cooking and combustible gases. As well as the treatment of drinking water and sanitation and ability to prevent infections, reduction of emissions that result and pollute from non-clean energy sources utilization, the adoption of environmental materials and assets, while making clean indoor environments and reducing exposure to hazardous remains. Moreover, according to world health organization (WHO) in 2018, air polluted household environments are responsible for 3.8 million deaths annually. Usually, SHS happens for occupants under the age of 20 as they stay more at homes, where in office buildings its referred to as a sick building syndrome (SBS), with similar symptoms, that takes place but for older age groups ranging from 20-60 years old. In office buildings the occupancy levels are larger than in households, thus, it is easier to identify the causes of SBS than SHS. The study also investigated different research efforts from 1984 to 2010, indicating that yet all studies were dispersed in approach and limited over different geographical regions, such as: Europe, US, China, Brazil, and Egypt (Kishi and Araki, 2020).

Vilčeková et al. (2020) investigated the lifecycle assessment (LCA) of wooden housing units against short time measurement of IEQ indicators. Physical parameters were investigated such as temperature, relative humidity, air circulation and acoustical performance; the monitoring included measuring $\mathrm{CO}_{2}$ concentration (expressed in kilograms per space) and other volatile and non-volatile compounds. The findings highlighted that wooden houses with zero energy characteristics are considerably green buildings that have potential of improved IEQ conditions. The study linked the production of raw materials used in construction of wooden houses, to the environmental aspects, as a potential for achieving a high quality IEQ. The measurements taken, utilized different instruments, and sensing devices, that can provide quantified data for further analyses of the units' performance. The limitation of this study is that it focused on collection of measurements during short period of time (cold period of the heating season). The study indicated that wooden houses possess satisfactory IEQ performance conditions for its occupants.

Asojo, et al. (2020) conducted a POE on 2,275 occupants at 30 different workplaces, located at Minnesota, USA. The study indicated that male occupants were more satisfied of the thermal conditions of the workplaces, than their female counterparts. The study was performed on ranging group of occupants within the facilities who experienced a span ranging up to 9 years of time. The findings indicated that those occupants who stayed for 2 years or less periods were more satisfied about the environmental conditions, than those who occupied the work facilities for periods longer than 2 years. The elements investigated were related to different IEQ aspects, such as acoustic quality, ability to hear desired sounds, ability to limit noise, aesthetical appearances, quality of cleaning and maintenance, accessibility to and amount of daylight, illuminance performance, adjustability of ambient and task lighting, ergonomics of furniture, privacy, indoor air quality (IAQ), technological readiness, humidity, vibrations and view conditions. Consequently, the study recommendations aimed to inform designers of workplace facilities about considerations for IEQ, that may lead to satisfactory future occupation. The study was limited to subjective means of satisfaction and lacked the measurements of IEQ due to the physical buildings' characteristics.

Hameen et al. (2020) aimed to investigate IEQ performance in educational facilities, using the national environmental assessment toolkit, of USA. The research included measurements and satisfaction surveys collected from eight schools, over a period of eight years' time. The research highlighted the need to distinguish the post occupancy evaluation and measurements of school facilities, from workplace facilities. The research indicated that there is a lack of implementation of POE studies and measurements of IEQ in educational facilities, on a national and international level. Different types of data were collected ranging from excel sheets, 
photos, videos, interviews narrative, MySQL, and hand sketches. The IEQ elements under study were: glare on computer screens, audio visual discomfort, illuminance, optimal air quality, adequacy of monitoring devices, assessment of air temperature and movement, relative humidity, radiant temperature, acoustic attenuation, background sound levels and spatial qualities. A report was provided by the end of the study, listing every categorized element of IEQ against its performance with evaluation recommendations. The findings of this research allowed stakeholders of the educational facilities, to make informed decisions, about allocation of resources and investments toward the improvement of their facilities.

Romero Herrera et al. (2020) investigated the impact assessment reporting of IEQ comfort elements in two different office buildings, that have varying organizational cultures. The facilities were equipped with indoor environmental devices, and sensors that were used to collect, and analyze indoor climatic conditions. The study was conducted over 2 weeks. The study findings led to recommendations about the effective reporting reliability, of thermal comfort aspects. The recommendations highlighted the need for continuous monitoring of comfort elements, and the facility performance, over longer periods, to avoid simplification of findings and to foster contextual understanding of complex occupational behaviors, in facilities. The authors indicated that "A major shortcoming of most commonly used comfort parameters and scales is that they are based on lab research while often applied in real-world setups". Thus, the research indicated that there is a need to focus efforts on the role of ICT in supporting effective measurements and data capturing techniques in a systematic manner, serving the comprehensive need of understanding built indoor environmental performance; in link with energy use. The research also concluded, the need for avoiding averaging IEQ comfort related aspects, as applied by many of the short-term studies, trying to understand IEQ performance in buildings. The study utilized a specific mobile application, to collect users' subjective indicators, along with an accustomed sensor box to take field measurements, around the under-study facilities. The reports produced (from the analyses of collected data) were then used to assess comfort elements within the facilities under investigation.

Martin et al. (2020) utilized a benchmarking online platform, used to analyze POE data from 11 different higher education classrooms, as a survey collected from 3140 students, over 9 years, at Minnesota, USA. The study indicated the importance of specific IEQ elements, in relation to the gender of students. Yet, the research concluded the need for a benchmarking tool, that extends physical measurements along with the objective survey responses, from the students. Stating that benchmarking automation needs to be unified universally to allow for the better understanding and comparison of specific facilities performance under equivalent categories.

Valderrama-Ulloa et al. (2020) conducted a systematic literature review, on more than 100 IEQ studies, over different regional contexts. This is to synthesize the domain coverage and typologies of facilities which are lacked in IEQ research. The findings indicated that Argentina and Brazil fronted other countries in number of IEQ research studies, by contexts on residential and educational facilities. The study identified gaps in IEQ research, based on facilities types, such as nursery schools, senior homes, and health facilities. The study recommended the need for more IEQ research, to improve the regulatory standards and design of future facilities. As well as to improve operation, maintenance, and management of existing facilities. The alarming finding of this review is that different studies, comprising a total of 88 facilities in Latin America indicated that $67.5 \%$ of occupants are uncomfortable with their IEQ.

Larsen et al. (2020) developed an innovative tool, for the holistic evaluation of IEQ, to facilitate a graphical understanding and reporting of a facility IEQ performance. The tool comprised of elements such as: potential indoor air quality (IAQ), thermal, visual, and acoustical qualities. The holisticness of the tool can be set under question as it did not consider users subjective influences, as well as only 16 parameters that can be measured physically, but practically easy to measure. The parameters are then weighted to provide an overall rating indicator for IEQ of the built facility, in letters ranging from A to G, next to a scale of percentage scores. The tool assists facility managers and designers to identify IEQ problems and warrant about aspects for future design. The research did not introduce the potential for adding further parameters, yet the tool is considered as an innovative representation that can be replicated for reporting facilities IEQ performance. 


\section{A FRAMEWORK FOR THE MANAGEMENT OF IEQ IN BUILT FACILITIES}

A framework has been developed utilizing Integrated Computer Aided Manufacturing Definition for Function Modeling $\left(\mathrm{IDEF}_{0}\right)$ graphical modelling technique to represent the processes involved in the holistic management of IEQ, in built facilities, while supporting the needs for sustainable monitoring and update of IEQ indices. The framework assigns responsibilities, while inform on constraints that must be considered for the implementation of the processes. The framework comprises of 5 main processes: measure IEQ indicators, store data for IEQ indicators, Evaluate IEQ indicators, Update IEQ indicators, and implement the corrective measures. Each of the processes have multiple inputs to perform each of its underlying activities, and outputs as outcomes. The processes can be expressed, as follows (as shown in Figure 1):

\section{1 (P1) - Measure IEQ Indicators}

This process aims to uphold all the functions related to identifying the built facility characteristics, ensures identification, running communication to the physical measuring devices, sensors, and wearables of occupants. To initiate the collection functions of measured raw data in quantitative means, in forms of a big multi-dimensional table, for identified set of IEQ elements, as attributes. Inputs of collectors as data should be time stamped and logged in real-time. The output will be a big table of keyed raw data on a shell of dimensions. This process will be constrained by costs of the hardware, and communication infrastructure that will be invested in the facility and human gadgets in context. Table 1 enlists the proposed inputs for the system.

\section{2 (P2) - Store Data for IEQ Indicators}

This process aims to ensure the security and storage of data and serves to send the facility data, from its local storage, from an edge to a cloud end. This serves as a terminal for categorization of raw data into specific tables, for classification of data types based on units of measure, and facilities space attributes. This process must ensure the quality for validity, consistency, accuracy, timeliness, and completeness of data. The output will be a structured batch of data. This process is constrained by time stamp, where it is necessary to uplink to cloud continuously without latency.

\section{3 (P3) - Evaluate IEQ Indicators}

This process aims to analyze IEQ structured data to report the IEQ indicators and create meaningful insights about the facility's indoor environmental performance. The process inputs of structured data allow a bigger pool for indoor environmental quality categorization. Relations will be formulated between different attributes to calculate specific IEQ indicators. The users, on demand, may define the relationships between different attributes of data, to generate insightful graphs that can provide informed decisions about the operation and maintenance of the facility. These relationships can be accustomed and saved as coded queries, this can be referred to as the built facility intelligence reporting, where prediction analysis of IEQ can then take place based on the frequent queries of reports. The evaluation will be constrained by the national sustainability regulations, and time stamp for submission of reports. The output of this process is a batch of IEQ indices, that are analyzed with a status statement based on occupants' feedback. A weighted 5-points Likert scale can be utilized ranging from Satisfactory to unsatisfactory, to solicit the users' feedback. This process will be constrained by the time stamp, where cloud analytics should be run continuously without latency, to ensure that the reporting dashboards are updated with minimal processing latency.

\section{4 (P4) - Update IEQ Indicators}

This process allows the facility team to update their newly discovered IEQ indicators to a server of standardized IEQ indices, while at the same time ensures that the facility indices are updated to a national standard of IEQ. The output will be a request to update indices through detailed documentation of needed data and physical devices of measure, this is combined with a description of its underlying analyses. This process should be coordinated between the facility team and a sustainability team that represents a national authority at the other end, its responsibility to maintain quality sets of unified IEQ indices. 


\section{5 (P5) - Implement Corrective Measures}

This process serves to inform the facility management teams about the needed corrective measures prior for producing a comprehensive IEQ benchmark report. The functions involves a system ability to store and revoke recommended actions, based on the IEQ indices that were evaluated, the ability to control operation and maintenance tasks and report the action taken by the team within the facility. The process is controlled by operation and maintenance $(\mathrm{O} \& \mathrm{M})$ schemes, being cyclic or noncyclic O\&M to implement the corrective measures. The corrective measures are also constrained by the sustainability measures governing the facility. The output of this process is going to be a standardized report of categorized data schema that can be classified based on the built facility characteristics and profile.

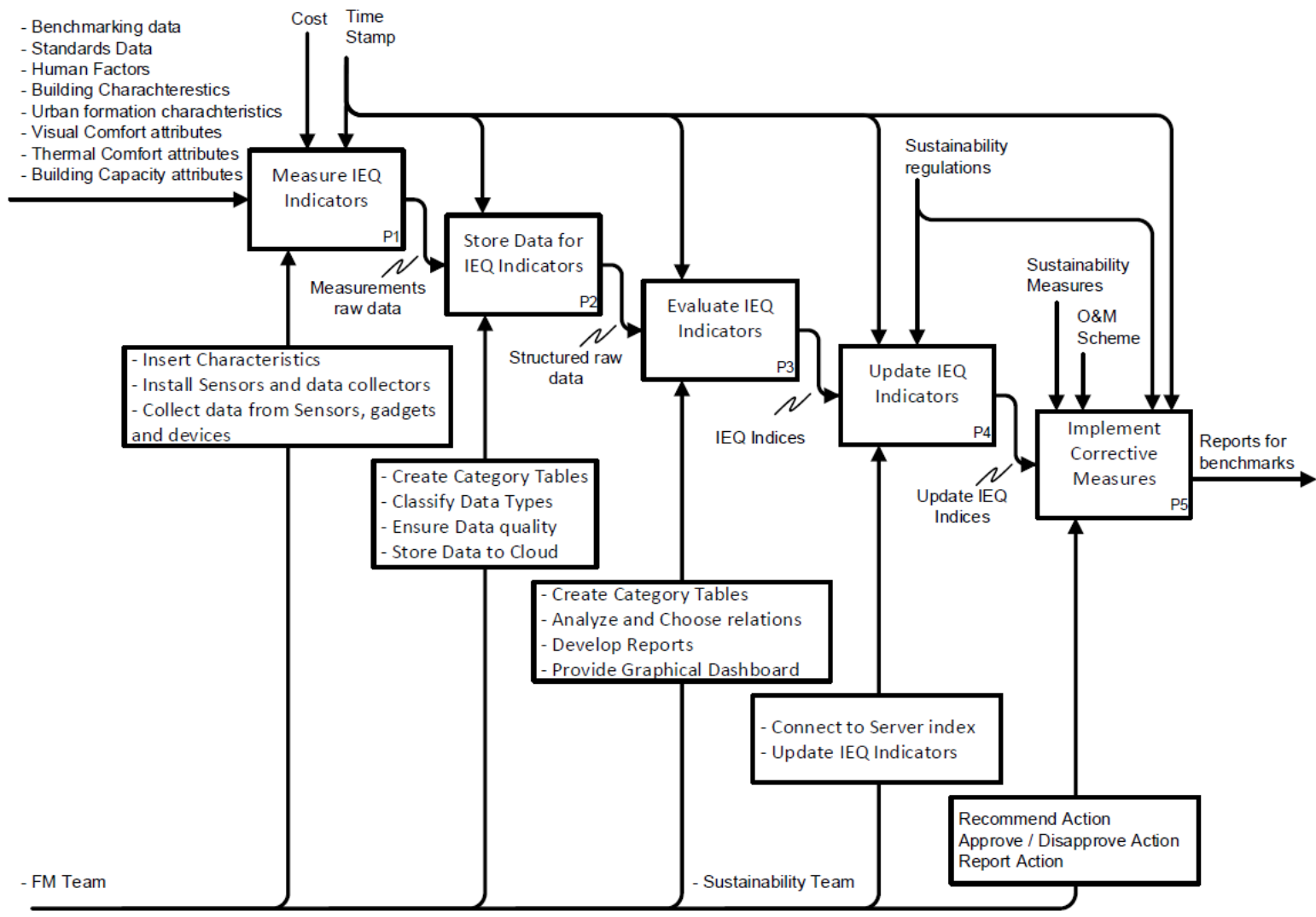

Figure 1. An IDEF $_{0}$ framework for the management of IEQ in built facilities

\section{DISCUSSIONS AND CONCLUSION}

The proposed framework introduces a systematic process for the holistic management of IEQ in built facilities. The framework utilizes $\mathrm{IDEF}_{0}$ modelling technique to ensure the inclusion of several activities, inputs, outputs and identified constraints that can be managed by different stakeholders. The emergence of internet of things (IoT), machine learning and artificial intelligence applications, serve the desired need for analysis of a universal IEQ database. There are variety of opportunities and potentials from collecting big data of built facilities performance and occupants perspectives, such as: 1) enabling researchers to generate and analyze accustomed relations between different attributes, at almost any locality, with ultimate enablement of classification and categorization of occupants' demographics and functional typologies of facilities, this can be expanded to micro climatic aspects as well as urban formulation impacts on indoor environmental qualities, 2) its universal implementation will allow for the interoperability of IEQ sensors and measuring devices by accommodating different inputs and output formats of data, 3) the framework uncovers the potential for starting a global open 
benchmarking tool, by proposing the use of accustomed dashboards, 4) the framework calls for focusing efforts on reducing costs of measuring devices and improving connectivity for ease of data collection, by introducing a management process, 5) the proposed framework implementation will serve urgent needs toward the realization of sustainability development goals (e.g. SDG 3 of maintaining good health and wellbeing, SDG 5 of gender equality, SDG6 of clean water and sanitation, SDG7 Affordable and clean energy, SDG 8 decent work and economic growth, SDG 9 industry, innovation and infrastructure, SDG 10 sustainable cities and communities, SDG 12 of responsible production and consumption and SDG 13 climate action) by allowing for a universal update of IEQ indicators and methods of calculation, 6) the implementation of the framework serves the need to maintain a historical record of IEQ performance of facilities, as many IEQ studies focused on the time dimension being a limitation of its findings, 7) the proposed framework allows for future integration of further processes; being dynamic in the making, 8) the framework widens IEQ management from being a research area to a job creation by integrating new mechanisms that can be applied on almost any type of facilities, 9) the framework supports the informed decision making upon operation and maintenance of facilities. Considerations for implementation of the framework include:

A. The development of platforms which serve its functions, must ensure user friendliness and unity of design.

B. The platforms must allow users to accustom the features and attributes that are needed for the analyses (Ioannou et al., 2019)

C. The implementation of socio-technical reporting techniques must serve the need for accuracy and representativeness of subjective voting and data collection for the active reporting of the occupants (Romero Herrera et al., 2020).

D. The machine learning capabilities based on the historical data and its characteristics, must allow for exploration of a large number of future scenarios, being predictive in approach.

More research is needed simultaneously with the implementation of the framework to experiment the impact of newly deployed collection devices, and sensors on real time performance. Furtherly a cost effective, precise, and secure modes of data communication need to be established for allowing transfer of a varying range of data types. The implementation of the proposed framework can allow for the graphical evaluation of complex scenarios using third-party computer simulations. Sustainability aspects are identified by the impacts of IEQ measures on global environment and earth simultaneously by green design of the built environment. The sustainability buildings standards such as Leadership in Energy and Environmental Design (LEED), Building Research Establishment Environmental Assessment Method (BREEAM), International Energy Conservation code (ICEC) and the American society for heating refrigeration and air condition engineers (ASHRAE) are all developed standards that can be synthesized by the sustainability experts world wide under the supervision of UN to globalize and ensure an international and wider aspect for implementation of the reporting processes. Leading technology providers can collaborate to develop a universal standardized code in collaboration with the UN and international standardization organization (ISO) to ensure a wider consensus of the framework and create an international definition book to ensure sustainability for the Triple Bottom Line (TBL) aspects from cultural, social and environmental perspectives; an emerging initiative such as Architecture 2030 could be an enabler for the implementation of the framework. Furtherly, the framework provides a process that engages sustainability experts in formulating codes by updating lessons learned, and concerns dealt with in professional practice; such link will adequately initiate a more effective development of standards and enhance realization of a sustainable built environment and future designs by evidence.

\section{REFERENCES}

Alexander, K. (1992). An Agenda for Facilities Management Research. Facilities, 10(7), 6-12.

Bae, S., Asojo, A. O., and Martin, C. S. (2020). Impact of occupants' demographics on indoor environmental quality satisfaction in the workplace. Building Research and Information, 48(3), 301-315.

Bae, S., Martin, C. S., and Asojo, A. O. (2020). Higher education students' indoor environmental quality satisfaction benchmark. Building Research and Information, 0(0), 1-16.

Bröchner, J., Haugen, T., and Lindkvist, C. (2019). Shaping tomorrow's facilities management. Facilities, 37(7-8), 366-380. 
Cao, S., Yu, C. W., and Luo, X. (2020). Indoor and Built Heating, ventilating and air conditioning system and environmental control for wellbeing. 0(0), 1-4.

Dan Norbäck, A. A. R. K. (2020). Indoor environmental quality and health risk toward healthier environment for all.

Danza, L., Barozzi, B., Bellazzi, A., Belussi, L., Devitofrancesco, A., Ghellere, M., Salamone, F., Scamoni, F., and Scrosati, C. (2020). A weighting procedure to analyze the Indoor Environmental Quality of a Zero-Energy Building. Building and Environment, 183(July).

Földváry Ličina, Veronika et al. (2018), ASHRAE Global Thermal Comfort Database II, Dryad, Dataset,

Geng, Y., Lin, B., and Zhu, Y. (2020). Comparative study on indoor environmental quality of green office buildings with different levels of energy use intensity. Building and Environment, 168(August 2019), 106482.

Hameen, E. C., Ken-Opurum, B., and Son, Y. J. (2020). Protocol for post occupancy evaluation in schools to improve indoor environmental quality and energy efficiency. Sustainability (Switzerland), 12(9).

Hassanain, M. A. (2007). Post-Occupancy indoor environmental quality evaluation of student housing facilities. Architectural Engineering and Design Management, 3(4), 249-256.

Heinzerling, D., Schiavon, S., Webster, T., and Arens, E. (2013). Indoor environmental quality assessment models: A literature review and a proposed weighting and classification scheme. Building and Environment, 70, 210-222.

Ioannou, L. G., Tsoutsoubi, L., Mantzios, K., and Flouris, A. D. (2019). A free software to predict heat strain according to the ISO 7933:2018. Industrial Health, 57(6), 711-720.

Kishi, R., and Araki, A. (2020). Importance of Indoor Environmental Quality on Human Health toward Achievement of the SDGs (pp. 3-18).

Larsen, T. S., Rohde, L., Jønsson, K. T., Rasmussen, B., Jensen, R. L., Knudsen, H. N., Witterseh, T., and Bekö, G. (2020). IEQ-Compass - A tool for holistic evaluation of potential indoor environmental quality. Building and Environment, 172.

Lindkvist, C., Temeljotov Salaj, A., Collins, D., Bjørberg, S., and Haugen, T. B. (2020). Exploring urban facilities management approaches to increase connectivity in smart cities. Facilities, ahead-of-print (ahead-of-print).

Ribino, P., Bonomolo, M., Lodato, C., and Vitale, G. (2020). A Humanoid Social Robot Based Approach for Indoor Environment Quality Monitoring and Well-Being Improvement. International Journal of Social Robotics.

Romero Herrera, N., Doolaard, J., Guerra-Santin, O., Jaskiewicz, T., and Keyson, D. (2020). Office occupants as active actors in assessing and informing comfort: a context-embedded comfort assessment in indoor environmental quality investigations. Advances in Building Energy Research, 14(1), 41-65.

Valderrama-Ulloa, C., Silva-Castillo, L., Sandoval-Grandi, C., Robles-Calderon, C., and Rouault, F. (2020). Indoor environmental quality in Latin American buildings: A systematic literature review. Sustainability (Switzerland), 12(2), $1-19$.

Vilčeková, S., Harčárová, K., Moňoková, A., and Krídlová Burdová, E. (2020). Life Cycle Assessment and Short-Term Measurements of Indoor Environmental Quality of a Wooden Family House. The 11th International Conference Environmental Engineering 11th ICEE Selected Papers, May, 21-22.

World Health Organization (2018). Household air pollution and health. Fact Sheets. Retrieved from URL: https://www.who.int/news-room/fact-sheets/detail/household-air-pollution-andhealth\#: :text=3.8\%20million\%20people\%20a\%20year,fuels\%20and\%20kerosene $\% 20$ for $\% 20$ cooking

Wei, W., Wargocki, P., Zirngibl, J., Bendžalová, J., and Mandin, C. (2020). Review of parameters used to assess the quality of the indoor environment in Green Building certification schemes for offices and hotels. Energy and Buildings, 209.

Yoseph, W. E.-S. (2020). Parametric Assessment for Achieving Indoor Environmental Quality (IEQ) in Egypt's New Urban Communities: Considering New Borg El-Arab City Urban Morphology and Openings' Specifications BT - Urban and Transit Planning: A Culmination of Selected Research P (H. Bougdah, A. Versaci, A. Sotoca, F. Trapani, M. Migliore, and N. Clark (eds.); pp. 87-100). Springer International Publishing. 